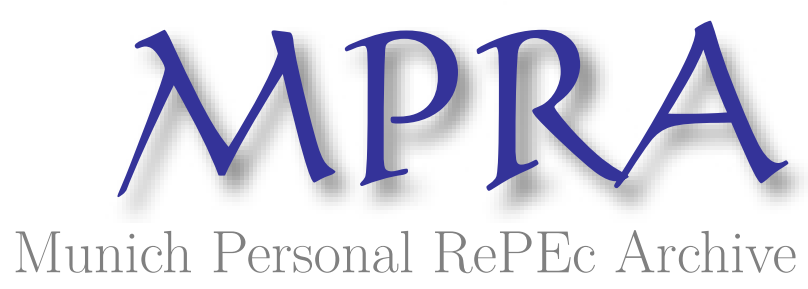

Analysis of average value of a Fourier series using z-transform: comparison with Hodrick-Prescott filter

Minskya, Ksovim

1 June 2016

Online at https://mpra.ub.uni-muenchen.de/71745/

MPRA Paper No. 71745, posted 04 Jun 2016 12:14 UTC 


\title{
Analysis of average value of a Fourier series using z-transform: comparison with Hodrick-Prescott filter
}

\author{
Ksovim Brkvyce Minskya
}

2016/06/01

\begin{abstract}
This paper develops a method of analyzing average value of a complexvalued function that can be represented as a Fourier series satisfying a few realistic restrictions. This method may be useful when Discrete Fourier transform is highly inefficient, and comparison with Hodrick-Prescott filter is made.
\end{abstract}

\section{Hodrick-Prescott filter}

Hodrick-Prescott filter has the following frequency response:

$$
H(\omega)=\frac{4 \lambda(1-\cos (\omega))^{2}}{4 \lambda(1-\cos (\omega))^{2}+1}
$$

as shown in [3]. The filter is high-pass filter - but one can convert it to become a low-pass filter, since the low-pass part that was filtered out can be obtained simply by subtraction.

The HP high-pass filter dependence on smoothing parameter $\lambda$ implies a need for decision: one can allow more low-frequency parts to be on data by increasing $\lambda$ and at first this decision seems good since the trend is obtained more clearly, but in many econometric usage, frequency is something related to error terms, and the linear trend at $\omega=0$ may not be the trend to be detrended.

Even if one wishes to obtain very close approximation linear trend, though, complexities around Hodrick-Prescott filter often does not allow it.

In this paper, though, I consider the case where one wishes to obtain exact linear trend. It is not practically possible for all time processes, but possible when $f(t): \mathbb{R} \rightarrow \mathbb{C}$ can be represented as fourier series involving finite harmonics, with other realistic assumptions on magnitude and negative/positive sign of individual amplitude of harmonics.

After performing analysis in z-transform, I consider a graph theory example. 


\section{Assumptions}

Any function mentioned in this paper is a function $\mathbb{R} \rightarrow \mathbb{C}$.

Definition 2.1 (Amplitude). For any arbitrary function $\alpha(t)$ expressible as $\alpha(t)=\sum_{\omega=-\infty}^{\infty} A_{\omega} e^{i \omega t / d}$ where $d$ is constant and does not vary with $\omega, A_{\omega}$ is said to be amplitude of $\alpha(t)$ at angular frequency $\omega / d$.

The function $f(t)$ to be discussed will be assumed to be expressible in Fourier series form - that is, angular frequencies with non-zero amplitude are all integer multiples of first harmonic angular frequency.

I will limit the number of angular frequencies with non-zero amplitude to be maximum of $n^{n}$, where $n$ may represent the input size, that is defined for each application of analysis developed in this paper. Also, the sum of all amplitudes will be restricted to maximum of $n^{n}$. But these limitations themselves is not central to analysis to be developed.

The central question to be answered in the analysis developed in the next section goes as follows:

If $f(t)$ is in the Fourier series form, has limited number of harmonics (and the maximum number of harmonics is known), and one can generate samples of $f(t)$ at time $t$, plus one can generate samples of $f(t)$ with frequencies scale such that new frequency $\omega^{\prime}=k \omega$, with $k$ constant, is there a good way to calculate amplitude of $f(t)$ at zero frequency? Discrete Fourier transform is there, but it may be very inefficient.

Definition $2.2\left(n_{h}, n_{p}\right) \cdot n_{h}$ is the amplitude of $f(t)$ at zero frequency. $n_{p}=$ $\sum_{\omega} A_{\omega}$.

\section{3 z-transform analysis}

From now on, angular frequency will be represented by both $\omega$ and $u$. Let the digital filter based on z-transform be:

$$
H(z)=\frac{1}{\left(z-p_{1}\right)\left(z-p_{2}\right)}
$$

where $p_{1}, p_{2} \in \mathbb{R}$ will later be defined. Let $\left|1-p_{2}\right| \gg|i u|$ and $\left|1-p_{1}\right| \ll|i u|$.

Definition $3.1\left(f_{1}, f_{2}, \mu_{1}, \mu_{2}\right) . f_{1}(t)=f(t)-n_{h}, f_{2}(t)=n_{h}, \mu_{1}(t)=e^{i u t}$, $\mu_{2}(t)=1$.

Definition $3.2\left(\gamma_{1}, \gamma_{2}\right) \cdot \gamma_{1}=1-p_{1}, \gamma_{2}=1-p_{2}$.

For input of $\mu_{1}(t)=e^{i u t}, \mu_{1}(z) H(z)$ is:

$$
\mu_{1}(z) H(z)=\frac{z}{\left(z-p_{1}\right)\left(z-p_{2}\right)\left(z-e^{i u}\right)}
$$


Taking inverse z-transform using each pole. For pole $p_{1}$, residue calculation results in:

$$
\frac{p_{1}{ }^{t}}{\left(p_{2}-p_{1}\right)\left(e^{i u}-p_{1}\right)}
$$

I will change the above to (subtracting away $t=0$ result):

$$
\frac{\left(1-\gamma_{1}\right)^{t}-1}{\left(-\gamma_{2}+\gamma_{1}\right)\left(i u+\gamma_{1}-\frac{u^{2}}{2}-. .\right)}
$$

For pole $p_{2}$, residue calculation:

$$
\frac{p_{2}{ }^{t}}{\left(p_{1}-p_{2}\right)\left(e^{i u}-p_{2}\right)}
$$

Changing to:

$$
\frac{\left(1-\gamma_{2}\right)^{t}-1}{\left(\gamma_{2}-\gamma_{1}\right)\left(\gamma_{2}+i u-\frac{u^{2}}{2}-. .\right)}
$$

For pole $e^{i u}$, residue calcaltion:

$$
\frac{e^{i u t}}{\left(e^{i u}-p_{1}\right)\left(e^{i u}-p_{2}\right)}
$$

Changing to:

$$
\frac{i u t-\frac{u^{2} t^{2}}{2}-\frac{i u^{3} t^{3}}{6}+. .}{\left(i u+\gamma_{1}-\frac{u^{2}}{2}-. .\right)\left(\gamma_{2}+i u-\frac{u^{2}}{2}-. .\right)}
$$

Now analyzing Equation 5, 7 and 9 by series-expanding based on $\gamma_{1}, \gamma_{2} a n d u$. Let $t=1$.

Definition 3.3 (Constant terms). Constant terms are the ones not depend on angular frequency $u$.

The focus will be on the constant term, more specifically on $\gamma_{1} / \gamma_{2}{ }^{2}$ terms. $\gamma_{2} \ll 1$ will be assumed. (which implies that $|i u| \ll 1$.)

First, Equation 5. The first initial term, based on the largest term in the denominator $-\gamma_{2} i u$ is:

$$
\frac{\gamma_{1}}{\gamma_{2} i u}
$$

Expanding series-wise, additive inverse of this term will be multiplied by the terms of the form $B /\left(-\gamma_{2} i u\right)$, where $B \neq-\gamma_{2} i u$ is some atomic additive term of the denominator in Equation 5.

Definition 3.4 (Atomic term). An atomic term of some equation refers to the term defined only by multiplication of positive powers of $\gamma_{1}, \gamma_{2}, u$ and constant $k \in \mathbb{C}$. Additionally, it is required that there should be no other atomic terms $j$ in the equation with $k=k_{j}$ such that only $k$ differ - in other words, $k=\sum_{j} k_{j}$. 
Definition 3.5 (Expanding factor). Expanding factor refers to $B /\left(-\gamma_{2} i u\right)$ in Equation 5, and for other equations, the denominator changes to the greatest atomic term in magnitude. By definition, any expanding factor is much less than 1 in magnitude.

Furthermore, additive inverse of these new terms will be multiplied by expanding factors to produce new terms, and this process continues indefinitely. Since our interest is in getting particular constant terms out of Equation 5, let us look back to the first dominant term in Equation 10. The denominator contains $u$ - in order to derive constant terms, some product of expanding factors that result in $D u / E$, where $D, E$ are only dependent on $\gamma_{1}, \gamma_{2}$ and some constant $k \in \mathbb{C}$.

Thus, either one directly starts off by multiplying the expanding factor that depends on $u$ (when fraction simplification is done) but only the first power of $u=u^{1}$, or one can multiply expanding factors that their product is either constant or depends on $u^{i}$ with $i \in \mathbb{Z}^{-}$then multiply by expanding factors that depend on $u^{j}$ with $j \in \mathbb{Z}^{+}$to obtain the final constant term.

First consider the greatest expanding factor in magnitude that depends on $u$, which is $-u /(2 i)$.

$$
\frac{-\gamma_{1}}{\gamma_{2} i u} \frac{-u}{2 i}=\frac{-\gamma_{1}}{2 \gamma_{2}}
$$

Since $\gamma_{2} \ll 1$, this term is much less than the constant terms being searched for.

Other expanding factors that depend on higher powers of $u$ are much less than $-u / 2 i$ in magnitude - thus, other directions that constant terms may be obtained do not have to analyzed.

Now to Equation 7. The initial dominant term is:

$$
\frac{-1}{\gamma_{2}}
$$

There is no expanding factor in this case that depends on $u^{i}$ with $i \in \mathbb{Z}^{-}$. Thus, for constant terms, one can restrict search to constant expanding factors. The only expanding factor is $-\gamma_{1} / \gamma_{2}$.

$$
\frac{1}{\gamma_{2}} \frac{-\gamma_{1}}{\gamma_{2}}=\frac{-\gamma_{1}}{\gamma_{2}^{2}}
$$

This is one of the terms being searched for.

Now to Equation 9. There are many initial terms:

$$
\frac{1}{\gamma_{2}}, \frac{-u}{2 \gamma_{2} i}, \frac{-u^{2}}{6 \gamma_{2}}, \ldots
$$

Let the first initial term be labelled as [1], the second initial term be [2], the third as [3].

Now to the expanding factors. First, the expanding factors that have dependency on $u^{-1}$ or are constant factors:

$$
\frac{\gamma_{1}}{i u}, \frac{\gamma_{1}}{\gamma_{2}}
$$


To eliminate $u^{2}$ in [3], one needs at least two $\gamma_{1} /(i u)$ multiplied, but the constant terms we are searching for only has one $\gamma$. Thus, [3] is ruled out. For [2], one needs at least one $\gamma_{1} / i u$ multiplied to eliminated $u$. However,

$$
\frac{u}{2 \gamma_{2} i} \frac{\gamma_{1}}{i u}=\frac{-\gamma_{1}}{2 \gamma_{2}}<\frac{\gamma_{1}}{\gamma_{2}^{2}}
$$

Thus, [2] is discarded also.

Now one is left with [1] only.

$$
\frac{-1}{\gamma_{2}} \frac{\gamma_{1}}{\gamma_{2}}=\frac{-\gamma_{1}}{\gamma_{2}^{2}}
$$

The above is one of the terms being searched for. But there is one other way to obtain constant term. For the term being searched, there is only one $\gamma_{1}$, and thus $\gamma_{1} /(i u)$ expanding factor can only be used once. Furthermore, before simplification, any expanding factor has denominator of $\gamma_{2} i u$. Recall that expanding factor has form $B /\left(\gamma_{2} i u\right)$. After using one $\gamma_{1} /(i u)$, this means that the additional expanding factor can only have $B$ that does not depend on $\gamma_{1}, \gamma_{2}$ and dependent on $u^{2}$. The only $B$ that satisfies such a criterion is $-u^{2}$. Thus, two expanding factors together multiplied that can be used to obtain the desired constant term are: $\gamma_{1} /(i u)$ and $-u /\left(\gamma_{2} i\right)$.

$$
-\frac{-1}{\gamma_{2}} \frac{\gamma_{1}}{i u} \frac{-u^{2}}{\gamma_{2} i u}=\frac{\gamma_{1}}{\gamma_{2}^{2}}
$$

This completes our analysis for $\mu_{1}(t)$. Now summing up pieces: Equation 13, 14, 15:

$$
\frac{-\gamma_{1}}{\gamma_{2}^{2}}
$$

Now to $\mu_{2}(t)$. For input of $\mu_{2}(t)=1$,

$$
\mu_{2}(z) H(z)=\frac{z}{\left(z-p_{1}\right)\left(z-p_{2}\right)(z-1)}
$$

For pole $p_{1}$, inverse-z transform residue after subtracting away $t=0$ part:

$$
\frac{p_{1}{ }^{t}-1}{\left(p_{1}-p_{2}\right)\left(p_{1}-1\right)}
$$

taking $t=1$ and converting to $\gamma_{1}, \gamma_{2}$ form:

$$
\frac{-\gamma_{1}}{\left(\gamma_{2}-\gamma_{1}\right)\left(-\gamma_{1}\right)}=\frac{1}{\gamma_{2}-\gamma_{1}}
$$

For pole $p_{2}$, inverse-z transform residue after subtracting away $t=0$ part:

$$
\frac{p_{2}^{t}-1}{\left(p_{2}-p_{1}\right)\left(p_{2}-1\right)}
$$


$t=1$ and converting,

$$
\frac{-\gamma_{2}}{\left(\gamma_{1}-\gamma_{2}\right)\left(-\gamma_{2}\right)}=\frac{-1}{\gamma_{2}-\gamma_{1}}
$$

Pole 1 will not be considered, as $1^{t}=1$, and the residue therefore does not depend on $t$. Adding together Equation 19 and 21:

Now let us go back to $f_{1}(t)$ and $f_{2}(t)$. The principle remains exactly the same as with $\mu_{1}$ and $\mu_{2}$. But because of amplitudes, Equation 16 will change to:

$$
\left(n_{p}-n_{h}\right) \frac{-\gamma_{1}}{\gamma_{2}^{2}}
$$

Thus, now it is possible to obtain the value of $n_{h}$, since we already know what $n_{p}$ is.

\section{Applications: Graph theory}

I will use the analysis developed above for finding the number of hamiltonian paths: here $n_{h}$. Hamiltonian path models have recently been used to study Nash equilibrium, as in [4] and [2].

Definition 4.1 (Base- $n$ expansion). Base- $n$ expansion of some number $k$ is basically expressing $k$ in base- $n: k= \pm \sum_{p=-\infty}^{\infty} a_{p} n^{p}$ with $0 \leq a_{p}<n$.

The power of base- $n$ is that if important parameters are the finite sums (that is, $k= \pm \sum_{p=b_{l}}^{b_{h}} a_{p} n^{p}$, with $b_{l}$ and $b_{h}$ finite), instead of infinite sums, then analysis becomes much easier. For studying numerical approximation of $k$ (if exact value cannot be known), one can just focus on finite number of numerical digits.

Definition 4.2 (graph, $n$ ). A graph $G$ is denoted with $G=(V, E)$ as done in the standard literature. $n=|V|$ is assumed whenever $n$ appears.

Definition 4.3 (walk, $n$-walk, hamiltonian path). A walk is defined as in the standard graph theory vocabulary. A walk that has $n$ vertices is called $n$-walk. Let us represent a walk with a list (tuple) of vertices in a traversing order from the start vertex to the end vertex. By the definition of a walk, one vertex can appear more than once in a list. A hamiltonian path, as defined in the standard graph theory vocabulary, is a walk with $n$ distinct vertices, where $|V|=n$.

Definition 4.4 (vertex). A vertex is assigned a number. Each distinct vertex has a distinct number. Let $V=\left\{n, n^{2}, n^{3}, \ldots, n^{n}\right\}$. From now on, one can assume a vertex as a number whenever appropriate.

Definition $4.5\left(n_{h}, n_{p}\right) \cdot n_{h}$ is the number of hamiltonian paths of $G . n_{p}$ is the total number of $n$-walks of $G$. 
Definition 4.6 (Vertex-number). The vertex-number of a walk is defined as the sum of all elements (vertices) in the list of a walk.

Note that the vertex-number of a walk represents the angular frequency of a walk in $x(t)$, as will be seen. It is certainly possible that two walks may occupy the same frequency. If there are $k$ walks that occupy the same frequency $\omega_{a}$, then the amplitude at the frequency would be $k$ in Fourier series language, or $k \delta\left(\omega-\omega_{a}\right)$ in Fourier transform language where $\delta(\omega)$ is a dirac delta function. The maximum number of vertices inside a walk is restricted to $n$, for sake of convenience.

Definition 4.7 (Permutation of a list). A permutation of a list is a re-ordering of list elements in $\xi$. For example, for $\xi=\left(\xi_{1}, \xi_{2}, . ., \xi_{n}\right), \xi_{\alpha}=\left(\xi_{n}, \xi_{n-3}, \xi_{n-4}, \ldots, \xi_{1}\right)$ is a permutation of $\xi$.

Lemma 4.1. Given $V$ as defined above, a vertex-number can only be formed out of a permutation of a single vertex-number list.

Proof. The proof is simply the basis representation theorem, where basis are elements in $V$. One exception to this proof, though, arises when a list $\xi$ representing a walk may be of $(k, k, \ldots k)$ with $|\xi|=n$ and $k=n^{i}$, or in words, there are $n$ k's in $\xi$. In this case, $n k=n^{i+1}$, meaning the vertex-number $\xi$ equals one of vertices in $V$. But this should not matter whenever walks one deals with have same number of vertices.

Following from above:

Definition 4.8 (Contribution of each $n$-walk to $x(t)$ ). From above, each walk has a vertex number $k$. Each $n$-walk is said to contribute $e^{i k t}$ to $x(t)$.

Definition 4.9 (Amplitude). For any arbitrary function $\alpha(t)$ expressible as $\alpha(t)=\sum_{\omega=-\infty}^{\infty} A_{\omega} e^{i \omega t / d}$ where $d$ is constant and does not vary with $\omega, A_{\omega}$ is said to be amplitude of $\alpha(t)$ at angular frequency $\omega$.

\subsection{Grid: $x(t)$}

Definition 4.10 (Grid, wires). A grid consists of $n$ depths, with each depth being equivalent to a column. Each depth contains $n$ vertices as in $V$. Each wire connects a vertex $v_{\alpha}$ from $i$ th depth to a vertex point of $v_{\beta}$ in $i+1$ th depth. A wire is connected between $v_{\alpha}$ to $v_{\beta}$ if and only if $\left(v_{\alpha}, v_{\beta}\right) \in E$.

Definition 4.11 (Function transmission: first depth case). In the first depth (first column), each vertex $v_{\alpha}$ transmits $e^{i v_{\alpha} t}$.

Definition 4.12 (Function transmission except for first and $n$th depth). Defining for each $v_{\alpha}$ in arbitrary $i$ th depth. All incoming wire transmissions $w_{\zeta}(t)$

from each wire $\zeta$ from $i-1$ th depth to $v_{\alpha}$ in $i$ th depth are summed, or equivalently $w_{\lambda}=\sum_{\zeta} w_{\zeta}$. And then multiply by $e^{i v_{\alpha} t}$ and transmit $u_{v_{\alpha}}=e^{i v_{\alpha} t} w_{\lambda}$ to each wire starting from $v_{\alpha}$. 
Definition 4.13 (Vertex point function transmission: $n$th depth case). All incoming wire transmissions $w_{\zeta}(t)$ from each wire $\zeta$ from $n-1$ th depth to $v_{\alpha}$ in $n$th depth are summed, or equivalently $w_{\lambda}=\sum_{\zeta} w_{\zeta}$. And then multiply by $e^{i v_{\alpha} t}$, resulting in $s_{v_{\alpha}}=e^{i v_{\alpha} t} w_{\lambda} \cdot x_{\text {ideal }}(t)=\sum_{v \in V} s_{v}$ is the output of the grid, not considering quantization errors involved.

For each depth $i, \sum_{v \in V} u_{v}$ shows the sum of all vertex-numbers representing $i$-walk.

\subsection{Post-grid: $y(t)$}

Simply, this post-grid procedure is all about calculating $y(t)=x(t) e^{-i \omega h t}$ where $h=\sum_{i=1}^{n} n^{i}$, the hamiltonian frequency of $x(t)$. Thus, $y(t)$ has 0 has hamiltonian frequency.

\subsection{Post-grid: $f(t)$}

$f(t)$ is defined as $f(t)=y(c t)$. $c$ will be defined later.

Let the angular frequencies of $f(t)$ be labelled with $u . u=0$ refers to hamiltonian frequency.

From now on, when it is said "every $u$," this refers to every $u$ with non-zero amplitude in $f(t)$.

\subsection{Sinusoidal quantization errors}

For every vertex $v$ of each depth of the grid, the numbers from maximum of $n$ vertices are added and then multiplied by $e^{i v t}$, for each $t$.

For each vertex $v$ of each depth, the error occurred would be of the following form:

- (Sum of errors from previous depths $) \times\left(e^{i v t}+\right.$ calculation error for $\left.e^{i v t}\right)$ + (The correct sum of previous depths) $\times$ (calculation error for $\left.e^{i v t}\right)$.

Here the purpose of error analysis is not to find out exact error but to derive the formula for the magnitude that is equal or bigger than actual possible maximum error.

Assume that the correct value of the previous depth is always $2 n^{n}>\left|n^{n}+n^{n} i\right|$, and the correct value of $e^{i v t}$ is $2>|1+1 i|$. This is bigger than it actually is, thus Equation 24 is an overestimate of the sum of errors.

Definition $4.14\left(e_{v}, e_{i}\right) \cdot e_{i}$ representing total maximum sinusoidal quantization error in magnitude occurring from depth 1 to depth $i$ of all vertices, and $e_{v}$ represents the maximum error in magnitude that occurs from calculating $e^{i v t}$.

Note that $e_{v}$ and $e_{i}$ represents entirely different things, and $v$ inside $e_{v}$ is not an index, unlike $i$, which is an index, in $e_{i}$. 
Thus, this will yield the following recurrence equation:

$$
e_{i+1}=n^{2}\left[\left(2+e_{v}\right) e_{i}+2 n^{n} e_{v}\right]
$$

Now let us simplify Equation 24 by the following substitutions:

$$
\begin{gathered}
\Lambda=2 n^{2}+n^{2} e_{v}, \Upsilon=2 n^{n} e_{v} \\
e_{i+1}=\Lambda e_{i}+\Upsilon
\end{gathered}
$$

Assuming that we start from $e_{0}=0$ (for sure, depth 0 does not exist, but this can safely be used), by geometric series formula,

$$
\begin{aligned}
e_{i} & =\Upsilon \frac{\Lambda^{i}-1}{\Lambda-1} \\
e_{i=n} \equiv e_{n} & =\Upsilon \frac{\Lambda^{n}-1}{\Lambda-1}<\Upsilon \Lambda^{n}
\end{aligned}
$$

Assuming that $e_{v}<1 / n^{2}$, we can assume that $\Lambda<3 n^{2}$.

To incorporate the errors occurring from further calculating $y(t)$,

$$
e_{i=n+1} \equiv e_{n+1}=\Upsilon \frac{\Lambda^{n+1}-1}{\Lambda-1}<\Upsilon \Lambda^{n+1}
$$

with $\Lambda^{n+1} \approx 3^{n+1} n^{2 n+2} \approx n^{3 n+3} \approx n^{4 n}$, assuming $e_{v}<1 / n^{2}$.

\section{Conclusion}

This paper presented an alternative way of deriving the average value of a complex-valued Fourier series with realistic restrictions in case Discrete Fourier transform becomes costly. The paper then presents a possible application of the analysis.

\section{References}

[1] Bahar E. (1972). "The indefinite Z-transform technique and application to analysis of difference equations", Journal of Engineering Mathematics 6 (2): $125-132$

[2] Bhat N. et al. (2004). "Computing Nash Equilibria of action-graph games", Proceedings of the Twentieth Conference on Uncertainty in Artificial Intelligence 2004 .

[3] King, R.G. et al. (1993). "Low frequency filtering and real business-cycle", Journal of Economic Dynamics and Control 17: 207-231

[4] Mavronicolas, M. et al. (2005). "A graph-theoretic network security game", WINE 2005: 969-978 
[5] Tsai, S. C., et al. (1983). "Application of the z-transform method to the solution of the wave equation", Journal of Sound and Vibration 19 (1): 1720 . 